\title{
An Overview of Localization Methods for Multi-Agent Systems
}

\author{
Muhammad Saim, Khalid Munawar, Ubaid Al-Saggaf \\ Electrical and Computer Engineering Department, Faculty of Engineering King Abdulaziz University, Jeddah, \\ Saudi Arabia \\ Electrical and Computer Engineering Department, Faculty of Engineering King Abdulaziz University, Jeddah, \\ Saudi Arabia \\ Electrical and Computer Engineering Department, Faculty of Engineering King Abdulaziz University, Jeddah, \\ Saudi Arabia
}

\section{ABSTRACT}

Localization of multi-agent systems is a fundamental requirement for multi-agent systems to operate and cooperate properly. The problem of localization can be divided into two categories; one in which a-priori information is available and the second where the global position is to be ascertained without a-priori information. This paper gives a comprehensive survey of localization techniques that exist in the literature for both the categories with the objectives of knowing the current state-of-the-art, helping in selecting the proper approach in a given scenario and promoting research in this area. A detailed description of methods that exist in the literature are provided in considerable detail. Then these methods are compared, and their weaknesses and strengths are discussed. Finally, some future research recommendations are drawn out of this survey.

Keywords: Localization, mu lti-agent, a-priori, survey

\section{INTRODUCTION}

$\mathrm{T}$ As societies have evolved over time, they have grown more complex and densely populated. With the advancement in science, the problems have also become more complex and so do their solutions. To solve complex problems, multiple autonomous systems must work cooperatively. Although the concept is not new but recent advancements in technology, particularly computation resources and communication, made it feasible to envision a large number of autonomous systems operating cooperatively.

Multi-agent systems are composed of multiple autonomous agents working cooperatively to achieve a task. Agents in a multi-agent system generally operate in close vicinity requiring a precise understanding of their individual locations to avoid collisions during navigation and to maintain connectivity and formation. Therefore, accurate, simpler and efficient localization methods are of great importance and there has been continuous research in this area.

Study of localization methods involving multi-agent systems can be largely categorized into two parts. In one category, the agents localize themselves using a-priori information such as the initial positions of the agents and the boundary of the environment or workspace etc. The second category, however, includes methods that do not require a-priori information but use statistical tools to predict the global positions of the agents; the agents possess sensors that can measure changes in their own motions and can detect other agents in their neighborhood.

The main goal of this study is to highlight the importance of this complicated and very much involved area of research through providing a comprehensive survey of the recent research in the field of multi-agent localization. This is to educate the researcher new to this area about the current state-of-the-art so that they can quickly approach a conclusion of what approach they should get into. In this way, this study will be very much helpful in promoting research in this area.

\section{BACKGROUND}

\subsection{Localization using A-priori Information}

A-priori information is the information that is derived based on observations rather than experiments. In the multi-agent scenario, a-priori information refers to the knowledge of initial positions of the agents and exact positions of landmarks in a global frame of reference etc. Most of the times, a-priori information is hard coded into an agent's control and guidance system. In this section, the methods which use landmarks to localize the agents are discussed in good details. Finally, a recently developed efficient method that does not require the knowledge about the landmarks is explained.

\subsubsection{Triangulation}

The most classical method of localization using landmarks is triangulation; it is perfectly 
applicable for the localization of multiple agents too. To understand this method, we need to consider an example in which a ship captain uses three landmarks to identify the approximate location of the ship in the sea. The captain knows the exact position of a landmark on a map and using a compass identifies the angle at which the landmark is located at a given moment on the map, and draws a line. The same procedure is repeated with other two landmarks too; the intersection region given the exact location of the ship. Ideally, all the three lines should cross at one point; but due to errors a triangle is formed, and the ship is anywhere in the region. In presence of uncertainties, this becomes a crude method of identifying the location of an agent, however it is still useful if the agents in the region do no come too close to each other. Various similar methods exist in the literature [4-6]. The accuracy of all these methods depends upon the number of landmarks, their distance with the agent, and the size of the environment.

There has been a lot of work on different improvements in the triangulation method. One such improvement considers two landmarks and utilizes the inter-agent distances; localization is achieved by calculating the angle subtended at each agent by lines drawn from two land marks at known positions and by computing the inter-agent distances [3]. Th is method is best suited for the vehicles which use GPS information for their own position acquisition; therefore, a loss of GPS signal is a serious issue. As an alternative to this approach which is especially suited for indoor environments where GPS information is totally unavailable, consider three UAV's trying to localizing themselves using two landmarks. As the GPS is unavailable, therefore the heading information is also missing. Nevertheless, the need for heading is compensated by using angle differences [7]. An angle will be subtended by each agent to the two landmarks; each agent will lie on a circle whose center will lie on the perpendicular bisector of the line joining the two landmarks. Multiple solutions can exist in this approach; for example, in case of three UAV's, there will be twelve (12) possible known solutions.

\subsubsection{Global Positioning System}

For global localization of agents in the outdoor environment, the Global Positioning System (GPS) is the most effective solution. This system works with the help of multiple geo-stationary satellites. The agents have compatible radio receivers that can receive signals from these satellites. The exact location of a satellite with respect to globe is always known; and the satellites send time stamps that are synchronized with the receiving device. By calculating the time difference between the signal sent and received, the receiver can form a circle in which an agent can be; a similar procedure is repeated with other geo-stationary satellites. The resultant position of a receiver is the intersection region of all these circles. The accuracy of this methods is usually in a few meters [1] and is therefore good enough if the agents do not come close to each other. However, in case of a close coordination among the agents, a much higher accuracy will be required. In such a scenario, the GPS cannot be utilized as a standalone tool for task achievement in multi-agent systems. Furthermo re, in indoor environments, this method becomes ineffective altogether [2].

\subsubsection{Nodes Emitting Microwave and Ultrasonic Pulses}

In this approach, the localization is achieved using the nodes that simultaneously transmit radio and ultrasonic pulses. By measuring the time difference between the received radio signal and the ultrasonic pulse, the distance of a node from an agent can be easily calculated. By fusing the similar information from the other transmitting nodes repeatedly, an estimate of the position can be obtained [8]. The advantage of using nodes is that they can be deployed and tested separately but their integration can be easily done at a later stage.

\subsubsection{RFID Tags}

Radio Frequency Identification (RFID) tags can also be used as landmarks. RFID tags with their own signature electromagnetic field are placed on the floor at known locations in an environment; RFID receivers are mounted on agents' underbelly. Whenever an agent passes over a tag and receives the signature it automatically corresponds it with a location already known to the new position to find out its orientation and velocity [9], [10]. Although it is a good solution for multi-agent coordination yet it is expensive because of the number of tags needed for localization. Position estimation required for coordination of agents in close range would mean that there must be frequent RFID tags across the floor. The technique is not very good from the dynamics point of view as the number of tags will slow down the detection cycle and put a significant barrier on the velocity of the multi-agent system. Furthermore, more cost will incur if the tag are required to have the ability to assess orientation too [11].

\subsubsection{Intersection Regions}

Solving the localization problem through finding intersection regions in which an agent is located is a very simple but interesting approach. The agents start with known initial positions. An agent has an omni-d irectional light transmitter and a set of receivers mounted on it; it also has a well- 
synchronized clock and is assigned to a specific time slot. The agent transmits a pulse only in its designated time while all the other agents are in receiving mode at that time. As there are multiple receivers, each receiver is fixed towards a known direction with respect to the agent's frame of reference. As soon as some receiver(s) mounted on an agent detect a light signal, and having the knowledge of which other agent should be transmitting in the current time slot, the direction (conical region) of the other agent is immediately known. Similarly, all the other agents will also mark the direction of the transmitting agent with respect to their current position and orientation. This process is repeated for all the agents; each agent transmits a light pulse in its own designated time slot while all the others detect it and mark its region of presence with respect to their current position/orientation. When a cycle is complete, the acquired information is exchanged through wireless communication lin ks of all the agents. Once an agent has received information about itself from all the other agents, and knowing the last known position of the other agents, it tries to find out a subset region that gives its own approximate current location. Combined with dead-reckoning, the accuracy can be further increased. This method is scalable and accuracy increases with the number of agents. The complexity of computing the intersection region is based on the shape of the region. If the identified regions are assumed as circles, then computation of the intersection region will be $\mathrm{O}\left(\mathrm{n}^{2}\right)$, while if the regions are triangular, trapezoidal or rectangular, the complexity will be approximately $\mathrm{O}(\mathrm{n})$. One such example scenario is discussed in [22].

\subsection{Localization without A-priori Information}

As it is not generally possible to get the exact and full information required for localization from the sensors available with the agents and in the environment, the need of statistical tools for estimation of the unavailable or incorrect information is of utmost importance. The research on statistical localization can be divided into two major categories; position tracking to correct dead reckoning errors with known initial positions, and global self-localization with no knowledge of initial position. The latter is obviously a much harder problem than the former one. A few such statistical estimation approaches are mentioned here.

\subsubsection{Kalman Filtering}

In this method, the agents know their own positions and those of their neighbors; the collective global state in an environment is obtained by fusing the individual state information from all the agents. This fusion is generally done through the Extended Kalman Filter (EKF). [13] Discusses such a technique in which distributed communication method is used for collective localization. It is to be noted that localization of an individual agents is done by the sensors mounted on it but the global localization is done after fusing the data from all the agents in the environment. It is observed that the error in position and orientation of the individual vehicles is reduced. The same technique is applied to a heterogeneous group of agents and achieved similar results. By heterogeneous, it means that the types of vehicles (agents) and the sensors used can be different [12]. If there are no errors and uncertainties in the sensory information and no problems with the communication systems, it becomes a trivial problem to solve. However, as it is not the situation and there can be sensory errors and delays and/or packet drops in communication, EKF can give better estimates and even can handle the localization problem during communication issues without divergence. It will keep giving a clear picture of the states of all the agents, as the agents not only can localize themselves but also their neighbors, the neighborhood information can be used to estimate the position as the whole group [14]. The major disadvantage of this approach is that it requires a lot of communication among the agents for successful operation, and increasing the number of agents will choke the network. Moreover, the computational resource required for each agent is also significant.

\subsubsection{Particle Filtering}

Particle filtering is another statistical method widely used for localization which performs better that Kalman filter-base methods in terms of complexity [20]. Foundations of particle filtering method are based on Monte Carlo Localization (MCL) [21]. The agents do not have a-priori information; the required information for localization includes bearing angles and relative positions acquired through the motion based sensors. In [20], range and bearing information is used and a detection model is designed using probability density function; this function is based on relative observations made by the sensors and taking the advantage of coordination. The advantage of using particle filter here is that any arbitrary distribution will work. This technique has much less complexity than the other statistical solutions proposed. It is also better scalable as the solution reaches an ideal particle filter when the number of agents goes to infinity; however, it does converge to a finite steadystate error when the number of agents is finite.

\subsubsection{Markov Localization}

Markov localization has been applied on single robot localization successfully [15-17]. So me work done in the literature is based on the grid-based 
topological representation of a robot's state space while others use sampling-based representation [18]. Sampling-based representation of state space is better because it covers a wide range of belief functions in real time. Belief functions are a way of representing a system when some uncertainty exists. This work is extended to multi-robots through detecting the presence of other robots by any robot. Another approach uses probability density function to model the robot's position estimates [19]. Exponential complexity is avoided by using factorial representation, and each robot has computing capability and maintains its local belief function. During localization, robots can detect other robots to determine relative position. The reliability of the detection routine is modeled by learning a parametric detection model from data, using maximu $\mathrm{m}$ likelihood estimator. These detections are introduced in the model as additional constraints, thereby reducing the uncertainty in localization estimate. This process has been applied on practical systems successfully and it is a good solution for indoor applications.

\section{COMPARISON AND DISCUSSION}

In this section, a qualitative comparis on of all the above-mentioned localization techniques for multi-agent localization is presented. Based on this comparison, it will be easier to select an approach for the scenario at hand. A hierarchical distribution of all the techniques discussed in this study are presented in Figure 1.

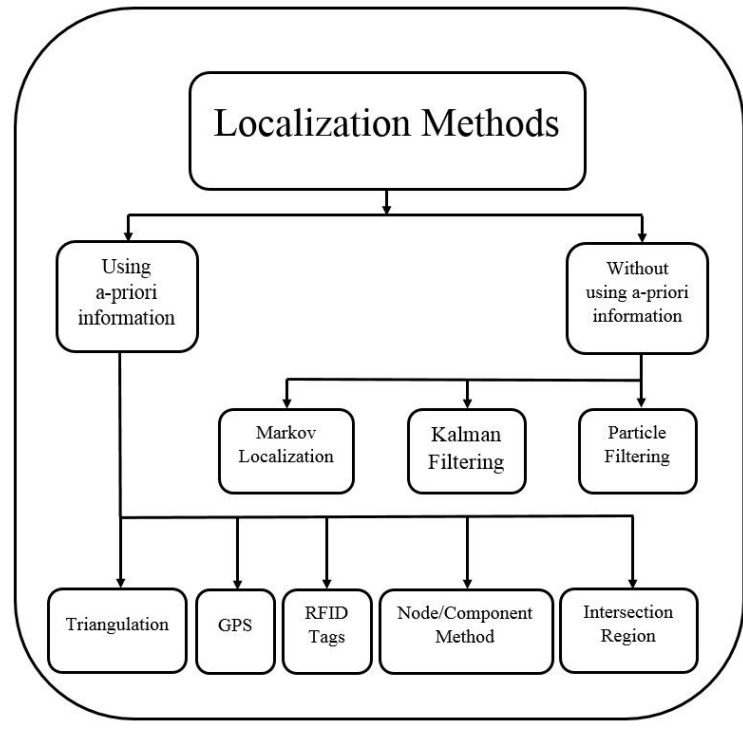

Fig 1. Hie rarchical dis tribution of the localization methods.

\subsection{Comparison of the Different Multi-Agent Localization Techniques}

The localization schemes for multi-agent systems mentioned in this study are compared here; this comparison is essentially of qualitative nature. Table 1 compares the localization techniques which require a-priori information while Table 2 compares those not requiring a-priori information. The different para meters compared are:

- Effectiveness; how quickly and correctly a technique can work,

- Computational complexity as referenced to the number of agents in the complete system,

- Applicability to indoor and/or outdoor scenarios, and

- Whether a technique works in a centralized fashion only or can also be applicable in distributed multi-agent systems.

TABLE 1 Comparis on of localization techniques requiring a-priori information.

\begin{tabular}{|c|c|c|c|c|}
\hline Techni que & Effecti veness & Complexity & Environment & Communication Topology \\
\hline Triangulation & Good & $\mathrm{O}(\mathrm{n})$ & Outdoor/Indoor & Distributed \\
\hline GPS & Good & $\mathrm{O}\left(\mathrm{n}^{2}\right)$ & Outdoor & Centralized/Distributed \\
\hline $\begin{array}{c}\text { Node/Component } \\
\text { emitting pulses }\end{array}$ & Very Good & $\mathrm{O}\left(\mathrm{n}^{2}\right)$ & Indoor & Centralized/Distributed \\
\hline RFID tags & Good & $\mathrm{O}(\mathrm{n})$ & Indoor & Distributed \\
\hline Intersection Region & Very Good & $\mathrm{O}(\mathrm{n}) / \mathrm{O}\left(\mathrm{n}^{2}\right)$ & Indoor/Outdoor & Distributed \\
\hline
\end{tabular}

TABLE 2 Co mparis on of localization techniques not requiring a-priori information.

\begin{tabular}{|c|c|c|c|c|}
\hline Technique & Effectiveness & Complexity & Environment & Communication Topology \\
\hline $\begin{array}{c}\text { Kalman } \\
\text { Estimation }\end{array}$ & Good & $\mathrm{O}^{2} \mathrm{n}^{2}$ & $\begin{array}{c}\text { Indoor/ } \\
\text { Outdoor }\end{array}$ & Distributed \\
\hline $\begin{array}{c}\text { Markov } \\
\text { Localization }\end{array}$ & Good & $\mathrm{O}^{2}\left(\mathrm{n}^{2}\right)$ & $\begin{array}{c}\text { Indoor/ } \\
\text { Outdoor }\end{array}$ & Centralized \\
\hline $\begin{array}{c}\text { Particle } \\
\text { Filtering }\end{array}$ & Very Good & $\mathrm{O}(\mathrm{n})$ & $\begin{array}{c}\text { Indoor/ } \\
\text { Outdoor }\end{array}$ & Distributed \\
\hline
\end{tabular}




\subsection{Discussion}

Triangulation is the most common, versatile and widely used localization technique in both indoor and outdoor environments; it has found its applications on sea, land, air and space. However, its accuracy greatly depends upon the precision of the knowledge of other agents and/or landmarks and the range/bearing measuring instruments. Therefore, it is not generally suited for close coordination among multiple agents. Among the modern techniques, the GPS-based localization is the most utilized and convenient method for localization of individual agents or multi-agent systems in the outdoor environments only. Even in outdoors, with weather variations and other occlusions, like thick clouds, there is a possibility of signal loss rendering the system ineffective; a good lock at least four satellites is an essential requirement for this system to start working. Therefore, other solutions must be considered for the situations in which GPS does not work. Node/component based method in which an agent emits pulses in different energy domains is also an effective method, however the data must be gathered at a single point for calculation hence making this tool less effective because of its limited scalability. The precision of localization using RFID tags is good especially for structured indoor environments but it is an expensive and a bit slower solution for localization. Calculation of intersection regions is although among the simplest techniques, yet it does not give an exact location of an agent but rather a region. Although, this intersection region becomes quite narrow when the agents come closer to each other, yet the help of other proximity sensors is crucially needs for direct interaction between the agents. This method can work in a scenario with a few obstacles even. Although it is a scalable method, yet increasing the number of agents will increase the length of a scan cycle resulting in slower performance.

Among the techniques not requiring the apriori information, the most commonly used techniques are the Kalman filter-based estimation techniques. Although the method has been improved a lot over the years and various variants like Extended Kalman Filter (EKF) have evolved, yet the complexity is still very high. Moreover, all these techniques essentially require a good knowledge about the agents being used and the sensors they possess; if there is a lack of knowledge about these, the Kalman filters will face serious convergence is sues. Markov localization, in comparison to Kalman filter, is better as a complete range of belief functions can be incorporated and modeled. But it also has a stringent requirement; all the information must be gathered at a single location for processing which makes the process unsuitable for distributed multi-agent systems because of a single point of failure and limited scalability. The particle filter based approach is among the most recently developed ones and applied successfully on multiagent systems; it has also reduced the complexity and can be applied in a distributed fashion. This approach is proven to be convergent in almost every possible scenario.

\section{CONCLUSIONS AND RECOMMENDATIONS}

Multi-agent systems are being employed for problems that are either impossible or too complex for individual agents to work with. Localization is one of the basic requirements for multi-agent systems for any type of objective they should accomplish. Localization process can be divided into two major categories; one requiring apriori information about the system and landmarks and the other working without it. With a-priori information available, there exist multiple reliable methods which have been widely used in practical systems. However, the problem of working with systems having no a-priori information is considerably more difficult and requires tools from statistics to draw results as there exist uncertainties and errors. This survey has briefly described each technique and mentioned its pros and cons. A qualitative comparison of all the techniques in both categories is also presented.

Among the techniques requiring a-priori information, triangulation is perhaps the simplest and most widely understood; most of the other techniques make use of some sort of triangulation. GPS based localization is among the most effective techniques for outdoor scenarios where a good satellite coverage can be achieved. Triangulation using bearing angle information lacks the accuracy required for agents coordinating closely with each other. Component emitting pulses of different wavelengths simultaneously is received, and fusion of multiple receptions is used to approximate the location, a reliable but centralized technique as all data has to be gathered at one place. RFID tags are expensive and place limitations on speed on which agents can operate. Intersection region method place limits on scalability because of its time cycle restriction.

Through the comparison and analysis of all these techniques, it is evident that the technique computing the intersecting regions is simple and effective. However, as it has scalability limitations, it can be recommended to modify it to work with only the neighbors' information; this will improve the scalability and effectiveness of the algorithm. In the component method, a few fixed nodes (beacons) can be established and all the agents will calculate their positions by fusing the information acquired from these beacons. Using this method, a more 
reliable and scalable distributed communication system can be realized.

Kalman filter based estimation has limited capability because of the limitation on its belief function. Therefore, it can be research opening to find out how to map any arbitrary belief function using this filter. Application of Markov localization techniques to distributed multi-agent systems will be of great interest; it will involve a question how to partition its pdf to suit such a scenario. Particle filtering, although the best among all the statistical approaches discussed, still has a lot of room for improvement to design more arbitrarily distributed agent detection models.

\section{REFERENCES}

[1]. R. Duffield, M. Reid, J. Baker, and W. Spratford, "Accuracy and reliability of GPS devices for measurement of movement patterns in confined spaces for court-based sports," Journal of Science and Medicine in Sport, vol. 13, no. 5, pp. 523-525, 2010.

[2]. A. El-Rabbany, Introduction to GPS: The global positioning system, Second edition, 2nd ed. Boston, 2006.

[3]. I. Shames, B. Fidan, B. D. O. Anderson, and H. Hmam, "Self-localization of mobile agents in the plane," $20083 \mathrm{rd}$ International Symposium on Wireless Pervasive Computing, 2008.

[4]. M. Betke and L. Gurvits, "Mobile robot localization using landmarks," IEEE Transactions on Robotics and Automation, vol. 13, no. 2, pp. 251-263, 1997.

[5]. I. Shimshoni, "On mobile robot localization from land mark bearings," IEEE Transactions on Robotics and Automation, vol. 18, no. 6, pp. 971-976, 2002.

[6]. H. Hmam, "Mobile platform selflocalisation," in Proceedings of Information Decision and Control, Adelaide, Australia, pp. 242-247, 2007.

[7]. H. Hmam, "Cooperative sensor selflocalization in the plane," submitted to IEEE Transactions on Aerospace and Electronic Systems, 2007.

[8]. B. Fidan and A. Camlica, "Least-squares based adaptive source localization by mobile agents," 2012 50th Annual Allerton Conference on Communication, Control, and Computing (Allerton), 2012.

[9]. J. L. Martinez Flores, S. S. Srikant, B. Sareen, and A. Vagga, "Performance of RFID tags in near and far field," 2005 IEEE International Conference on Personal Wireless Communications, 2005. ICPWC 2005.

[10]. V. Kulyukin, C. Gharpure, J. Nicholson, and S. Pavithran, "RFID in robot-assisted indoor navigation for the visually impaired," 2004 IEEE/RSJ International Conference on Intelligent Robots and Systems (IROS), 2004

[11]. S. Han, H. Lim, and J. Lee, "An efficient localization scheme for a differential-driving mobile robot based on RFID system," IEEE Transactions on Industrial Electronics, vol. 54, no. 6, pp. 3362-3369, 2007.

[12]. S. I. Roumeliotis and G. A. Bekey, "Distributed multirobot localization," IEEE Transactions on Robotics and Automation, vol. 18, no. 5, pp. 781-795, 2002.

[13]. A. Martinelli, F. Pont, and R. Siegwart, "Multi-robot localization using relative observations," Proceedings of the 2005 IEEE International Conference on Robotics and Automation, 2005.

[14]. N. Karam, F. Chausse, R. Aufrere, and R. Chapuis, "Cooperative multi-vehicle localization," 2006 IEEE Intelligent Vehicles Symposium, 2006.

[15]. W. Burgard et al., "Experiences with an interactive museum tour-guide robot," Artificial Intelligence, vol. 114, no. 12, pp. 3-55, 1999.

[16]. Konolige, Kurt, and Ken Chou. "Markov localization using correlation." Sixteenth International Joint Conference on Artificial Intelligence (IJCAI). Vol. 99. 1999.

[17]. D. Fox, W. Burgard and S. Thrun "Markov Localization for Mobile Robots in Dynamic Environments", Volume 11, pages 391-427, 1999.

[18]. F. Dellaert, D. Fox, W. Burgard, and S. Thrun, "Monte Carlo localization for mobile robots," In Proceedings of the National Conference on Artificial Intelligence (AAAI), 1999a.

[19]. S. Thrun et al., "MINERVA: A secondgeneration museum tour-guide robot," Proceedings 1999 IEEE International Conference on Robotics and Automation, 1999.

[20]. A. Prorok and A. Martinoli, "A reciprocal sampling algorith $\mathrm{m}$ for lightweight distributed multi-robot localization," 2011 IEEE/RSJ International Conference on Intelligent Robots and Systems, 2011.

[21]. D. Fox, W. Burgard, H. Kruppa, and S. Thrun. A probabilistic approach to collaborative multi-robot localization. Autonomous Robots, 8:325-344, 2000.

[22]. M. Saim, K. Munawar, M. Moinuddin, and U. Saggaf, "A Localization Approach in a Distributed Multi-Agent Environment," in Proceedings of the $6^{\text {th }}$ International Conference on Intelligent and Advanced Systems (ICIAS 2016), 2016. 